\title{
Cour d'animal : l'emploi des animaux pour établir le portrait de l'animalité chez les humains dans les œuvres littéraires et picturales à travers les âges
}

\section{Tina CHAN}

Depuis les débuts de la civilisation, il y a une division entre l'humain et l'animal autour de lui : l'animal est un être sauvage à domestiquer et à dompter, tandis que l'humain est un être intelligent - capable de penser et de capturer les autres espèces avec des cages et des fusils — et ainsi le dominateur. Néanmoins, étant donné que l'animal, marginalisé et réduit à un être impuissant, a été l'objet des premières peintures rupestres, l'inspiration des premières métaphores (Berger 7) — et a continué d'être adoré en tant qu'animal de compagnie à choyer — il est évident que, depuis son existence, l'humanité a entretenu des liens avec les animaux qui la fascinent. En particulier, les animaux sont souvent illustrés dans la littérature et dans les beauxarts; ce phénomène est particulièrement évident dans les œuvres suivantes: les tableaux de Pierre Paul Rubens du dix-septième siècle, les peintures hyperréalistes des années 1950 à 1990 de l'artiste canadien Alex Colville, le roman La rage de l'écrivain québécois Louis Hamelin, la pièce de théâtre québécois Tom à la ferme de Michel Marc Bouchard et dans La héronnière une collection de nouvelles québécoises de Lise Tremblay. Pourquoi les animaux fascineraient-ils les êtres humains s'ils étaient inférieurs ? Quels rôles jouent-ils dans les œuvres à l'étude?

Ces œuvres montrent que l'animal n'est pas inférieur, qu'il joue un rôle important et qu'il est doté de plusieurs facettes. En fonctionnant comme un sujet qui signale notre impuissance et nos faiblesses, un personnage innocent abusé ou un totem qui prête certains traits d'animalité à l'être humain, l'animal dans une œuvre démontre que l'humain n'est pas forcément plus raffiné que la bête comme la norme suggère, mais plutôt vulnérable, plein de malice et d'animalité. Autrement dit, les animaux dans les œuvres sont employés en contrepoint des humains, qui sont inférieurs et qui sont aussi animalesques que les bêtes autour d'eux.

Historiquement, les animaux ont souvent été vus dans la vie quotidienne soit comme des monstres à dominer et capturer, soit comme des créatures sous tutelle. Ce rôle semble inversé dans plusieurs œuvres littéraires et picturales : l'animal devient le maître (et parfois aussi le gardien). Par exemple, malgré le fait que les titres évoquent l'idée de la domination de l'homme, les tableaux La chasse au tigre et La chasse à l'hippopotame et au crocodile du peintre baroque 
flamand Rubens représentent l'infériorité évidente des humains dans la mise en scène. Dans ces scènes dynamiques de chasse, illustrant des bêtes attaquées par les humains qu'ils voulaient chasser, on observe la robustesse physique de l'animal qui domine l'humain. Malgré le fait que les autres animaux - comme le léopard mort et deux autres félins qui sont sur le point d'être tués - apparaissent vaincus dans La chasse au tigre, c'est le point focal, le tigre féroce du centre mordant l'épaule d'un homme à cheval, qui attire notre attention. La défaite de l'humain mordu est soulignée par l'emploi des couleurs plus vives que celles de l'arrière-plan. De plus, la fourrure orange du tigre s'oppose au corps bleu du cheval cabré qui, curieusement, n'a pas l'air d'être effrayé. En fait, la bouche fermée, il semble plutôt calme et coopératif, comme s'il voulait aider le tigre à se libérer des hommes. Dans La chasse à l'hippopotame et au crocodile, les animaux domestiques - les chevaux et les chiens de chasse - n'éprouvent aucune compassion à l'égard des animaux sauvages, mais c'est le point focal, l'hippopotame du centre, qui domine encore. Bien que la direction de la tête des chevaux mette l'accent sur le fait que les humains armés se trouvent derrière l'animal encerclé, la ligne de fuite passe rapidement de l'hippopotame à la tête du crocodile, l'autre protagoniste de la peinture selon le titre, en suivant la forme de sa queue. La position du crocodile souligne le visage effrayé de l'homme à gauche et sa bouche ouverte attire notre attention vers le chasseur blessé à sa droite. L'endurance et la résistance de l'hippopotame et du crocodile nous permettent d'espérer que les deux bêtes ne seront pas soumises à un terrible destin. Le regard terrifié de l'homme à côté du crocodile nous fait penser à l'échec imminent des chasseurs. Il faut donc reconnaitre que les animaux du centre des deux peintures, le tigre, l'hippopotame et le crocodile, ne se trouvent pas entièrement sous le sort de l'homme - ils sont plutôt victorieux. Ainsi, l'emploi des animaux confirme la fragilité et la vulnérabilité de l'homme. 


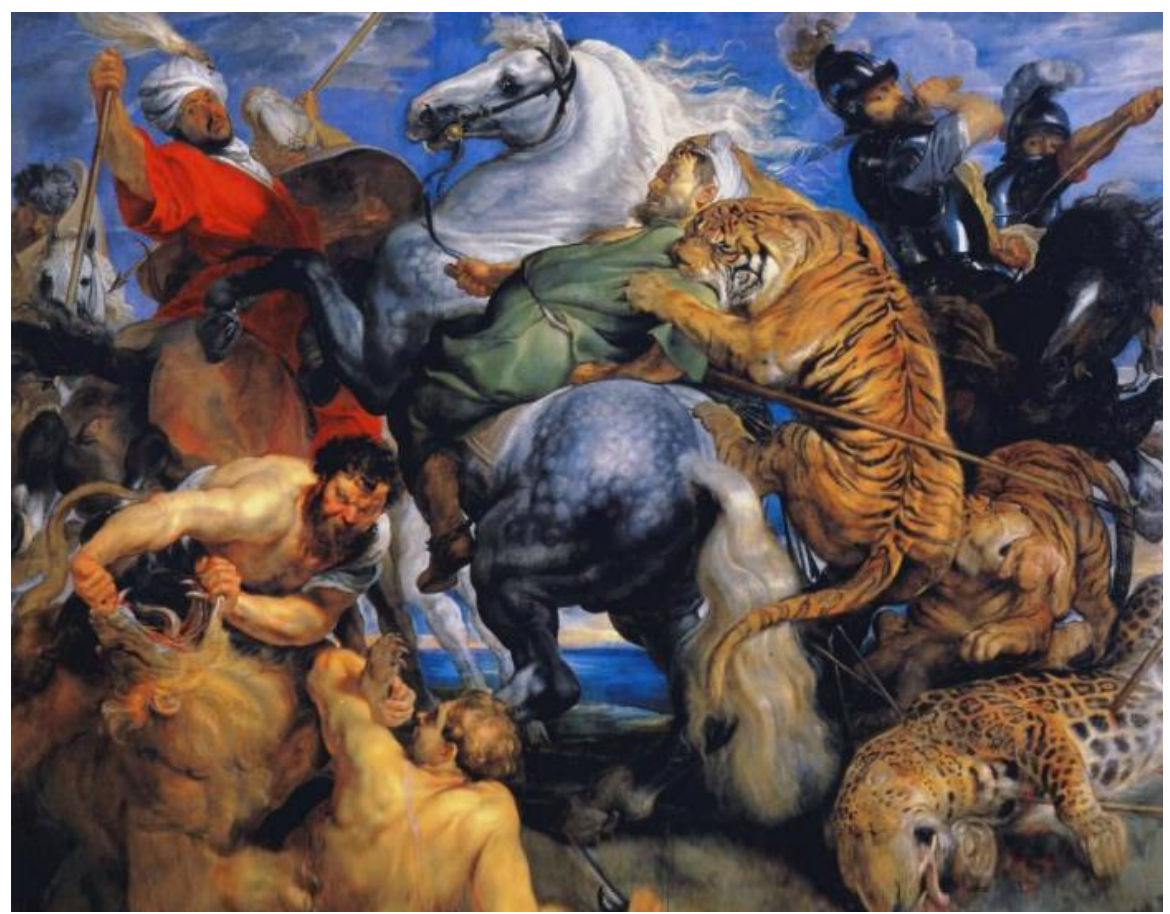

Pierre Paul Rubens, La chasse au tigre, 1617-1618, dans le domaine public.

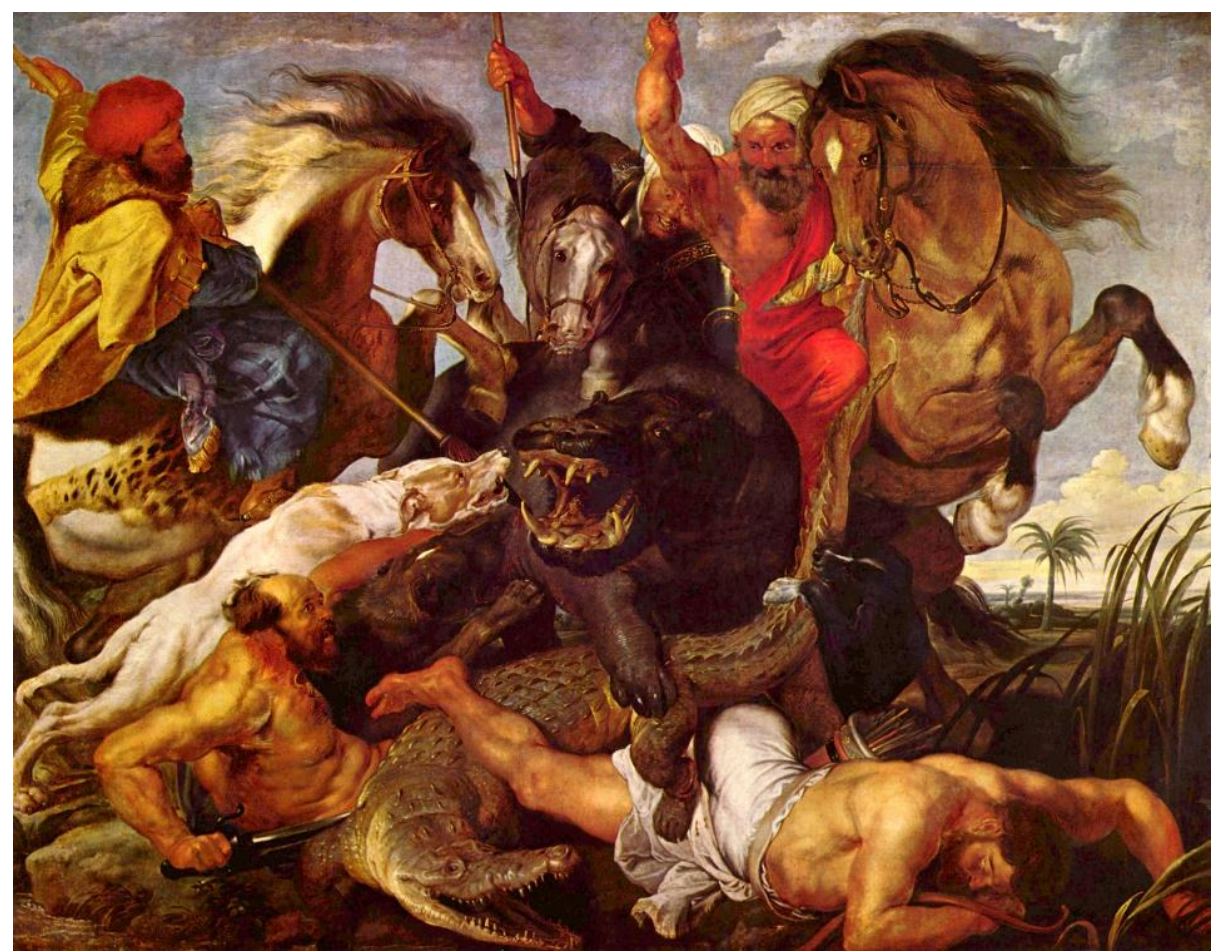

Pierre Paul Rubens, La chasse à l'hippopotame et au crocodile, 1615-1616, dans le domaine public. 
Ce phénomène se réaffirme dans les œuvres de l'artiste canadien Alex Colville. Ses tableaux hyperréalistes représentent des animaux pendant la deuxième moitié du vingtième siècle. La peinture intitulée Enfant et chien exemplifie en particulier son admiration pour les animaux de garde. Dans cette peinture, un chien noir aux yeux terreux et portant un collier clouté (qui lui donne l'impression d'être féroce) apparaît à côté d'un enfant nu et vulnérable qui, selon l'artiste, est censé être sa fille. L'enfant est aussi pâle qu'une poupée de porcelaine fragile, tandis que le chien, «protecteur des enfants », est solide et robuste avec ses griffes et son « regard hardi » («Animaux - Alex Colville - Musée des beaux-arts du Canada »). Selon les propos de Colville, il est «impossible [pour l'observateur] de ne pas ressentir un certain inconfort devant notre impuissance [en tant qu’êtres humains] » («Animaux - Alex Colville - Musée des beauxarts du Canada »). Certainement, il est facile de comprendre cette impression. Après tout, les deux figures sont de la même taille, mais l'animal est plus grand. En prenant plus d'espace au premier plan que le petit enfant, le chien domine l'image. Enfin, la fourrure noire du chien, couleur souvent associée au pouvoir ou au mal, s'oppose à la blancheur délicate du bébé, totalement nu, la main posée avec précaution, pour présenter la bête comme un véritable adversaire inquiétant capable d'attaquer l'enfant mais qui choisit de le protéger. 


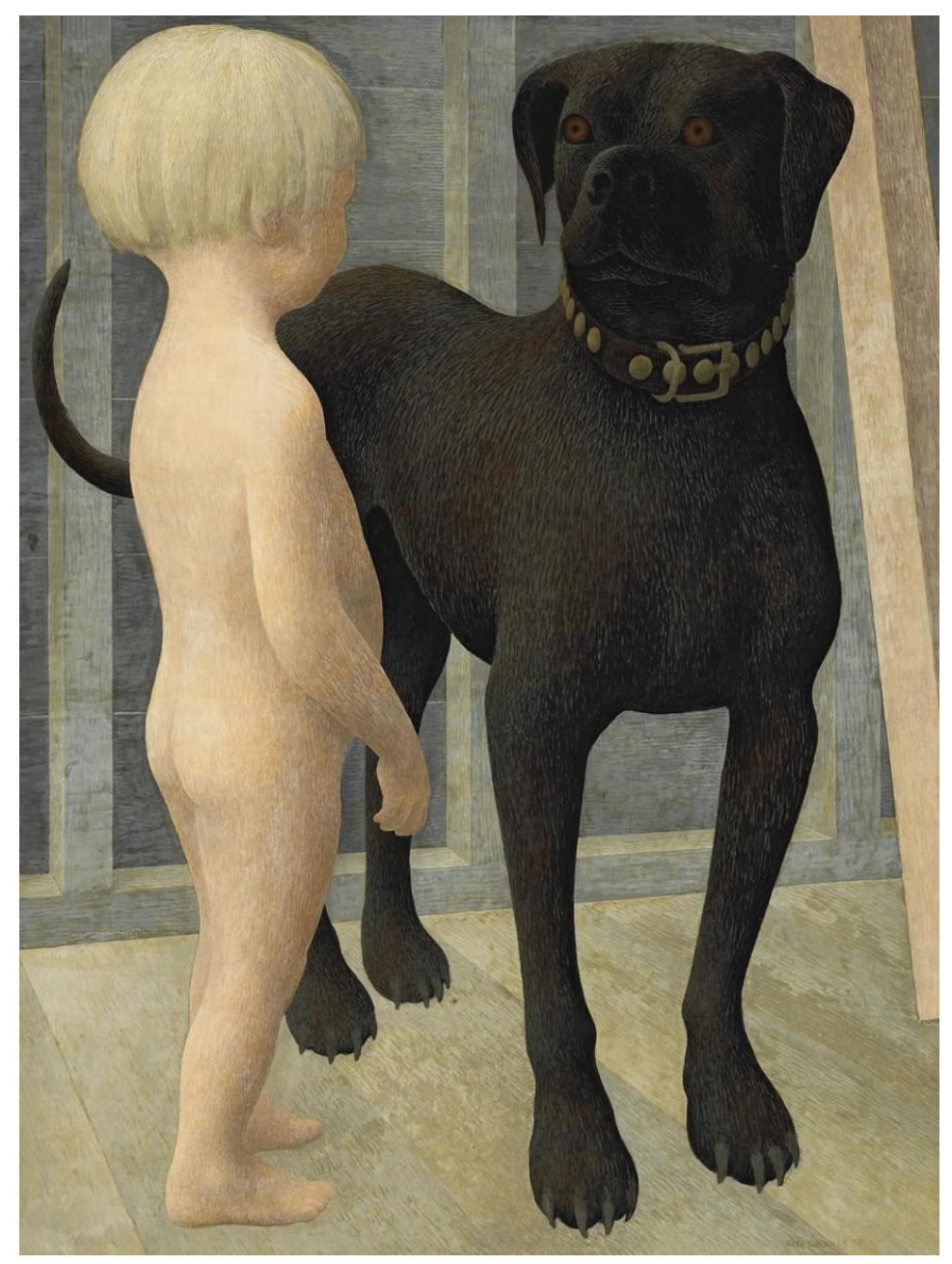

Alex Colville, Enfant et chien, 1952, Copyright A.C. Fine Art inc., tous droits réservés.

Ces œuvres montrent non seulement la puissance physique des animaux, mais aussi leur rôle en tant que maîtres des humains. L'humain est plus faible physiquement; les animaux sont leurs véritables protecteurs puisqu'ils ont besoin de leur présence et de leur aide pour se protéger.

Bien que les animaux soient souvent exclusivement illustrés de manière à faire ressortir leur férocité ou leur docilité, ils peuvent aussi être des personnages possédant leurs propres caractéristiques distinctives dans d'autres œuvres. Après tout, depuis la Renaissance, « $[\ldots]$ the association of animals with particular sins and vices was most common, but positive meanings were also transmitted by literary and artistic tradition » (Cohen 59-60). Plusieurs créateurs illustrent uniquement des traits positifs.

Colville est l'un d'entre eux : selon lui, il n'existe pas d'animaux méchants. En fait, il croit que les animaux, étant naturellement innocents, sont incapables de malice, pourvu que les humains ne les conditionnent pas pour agir autrement («Animaux - Alex Colville - Musée des 
beaux-arts du Canada »). L'idée de la pureté chez les animaux, selon Ann-Marie MacDonald, auteure de The Way the Crow Flies qui explore et critique le lien entre Colville et les animaux, vient de la croyance chrétienne que les humains sont les seuls êtres qui connaissent le péché. De plus, selon la comparaison de MacDonald, entre les animaux et les anges, les animaux font preuve d'une plus grande intelligence que les êtres humains grâce à leur instinct primaire et leur «programme sauvage » (wild agenda). L'homme devrait en faire autant. Par exemple, selon le Musée des beaux-arts du Canada, l'idée que les animaux peuvent voir « ce que l'être humain ne peut [pas] voir » est évidente dans Chien et toiletteur de Colville puisque le chien « regarde [l'observateur] droit dans les yeux ».

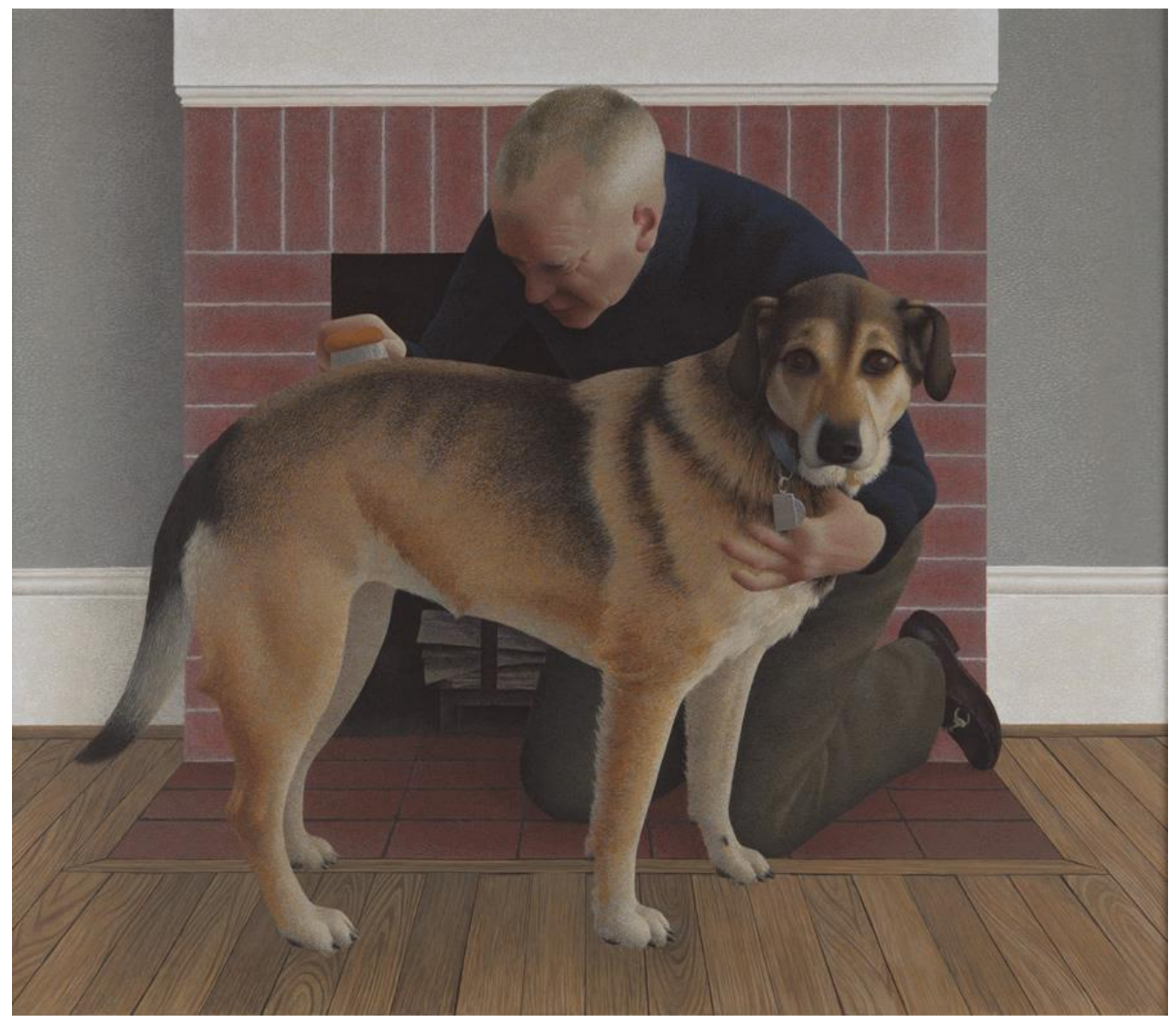

Alex Colville, Chien et toiletteur, 1991, Copyright A.C. Fine Art inc., tous droits réservés.

Pour cette raison, les animaux des peintures de Colville possèdent des qualités positives 
et angéliques, telles que sur la peinture Enfant et chien. Malgré le pelage noir du chien qui s'oppose à la pâleur de l'enfant et malgré l'apparence effrayante du chien, l'enfant n'a pas peur de lui, car le chien possède un regard innocent presque contemplatif, avec ses yeux écarquillés et ronds. Ce regard est encore visible dans les tableaux Chien et toiletteur et Chien et prêtre. Dans le premier, le chien est doté de sourcils détendus et cache ses crocs ; dans le second, la tête du chien noir remplace la tête d'un homme d'Église pour illustrer le prêtre, typiquement entendu comme un homme digne, bon et sage, comme le méchant dans la peinture — ou pour montrer que le chien est aussi pur que lui.

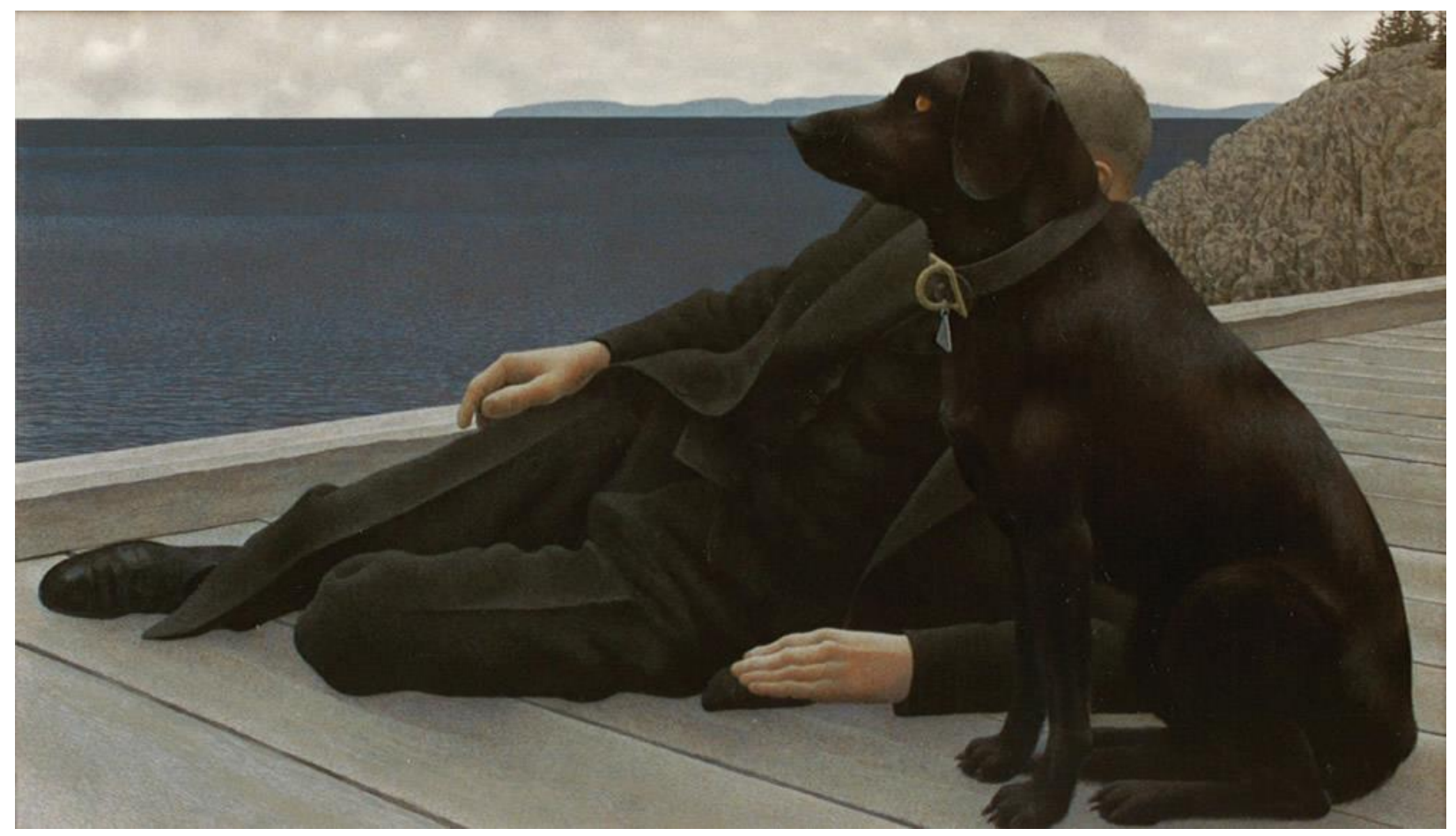

Alex Colville, Chien et prêtre, 1978, Copyright A.C. Fine Art inc., tous droits réservés.

Ce contraste de bienveillance s'observe aussi entre les humains et les animaux dans des œuvres littéraires, surtout sous la forme des coups de feu — c'est-à-dire sous la forme d'abattage. Par exemple, dans La rage de Hamelin, la chasse aux canards, à laquelle participent Édouard Malarmé et ses amis, est décrite comme une initiation guerrière : il faut se comporter en soldat ou en personne folle pour y participer. Après tout, selon Johnny, l'ami de Malarmé, « l'important, c'est de tirer. Il faut tirer, tirer, tirer [et puis] quelque chose va finir par tomber » (142). Cette mentalité effrénée ressort encore quand Johnny tire «mécaniquement », à bout pourtant, un lapin qui a un «cri trop humain » (297). Cette conduite s'oppose à l'inhumanité du tireur face à l'animal « trop humain ». Cela rend «Une éducation de l'instinct», le titre du 
chapitre qui contient la chasse aux canards, ironique. Malarmé ne reçoit pas « une éducation de l'instinct » : il perd plutôt sa rationalité que même les animaux, qui ne tuent pas pour s'amuser, possèdent. La perception de la chasse comme un jeu déraisonnable est comparée aux compétences que les chiens Hospodar et Icoglan possèdent à cette fin. Selon Malarmé, les chiens sont «inaptes à la chasse sérieuse des hommes » (139). Autrement dit, les chiens sont incapables de tuer déraisonnablement pour s'amuser et non pas pour manger, contrairement aux hommes.

L'abattage d'animaux innocents apparait aussi dans le recueil de nouvelles La héronnière de Tremblay. À plusieurs reprises y est décrite la mise à mort injustifiée d'animaux et aussi des « [mises] à mort de la proie [qui] n'a pas pour but l'alimentation» (Paré 464). Par exemple, Steeve, un adolescent dans un village québécois connu pour ses oiseaux indigènes et sa héronnière, tue plusieurs oiseaux en dehors de la chasse pour s'entraîner avec son fusil et se préparer au meurtre d'un homme qu'il déteste. Il méprise le fait que les victimes innocentes de sa colère, surtout les hérons qui n'ont rien à voir avec le motif de sa rage, sont des espèces protégées (Tremblay 25). Steeve souligne donc l'égoïsme des humains, qui favorisent leurs propres désirs sans considérer les autres autour d'eux.

Dans une autre nouvelle de l'œuvre de Tremblay, deux femmes trouvent un orignal au milieu de la route qui «avait été [pris] au collet et [...] [sectionné] en deux à la scie mécanique [par des braconniers] » (66). Cette description exemplifie à nouveau la persécution injustifiée des animaux par les humains. De plus, l'indifférence de Marius, qui ne veut pas dénoncer le crime des braconniers pour protéger la réputation et l'économie du village (70), confirme encore la favorisation de l'égoïsme et de l'avidité intéressée des humains.

Enfin, la femme de Marius, Élisabeth, maltraite des chatons avec son voisin. Ce dernier lance les pauvres félins «de toutes ses forces sur le mur de ciment devant lui » (62-63) sous le prétexte de «désinfecter l'étable » parce qu'il ne faut pas «laisser des chats proliférer sans contrôle » (63). Ce qui est le plus choquant dans cette scène, n'est pas forcément l'acte de tuer des animaux de compagnie innocents, sinon l'attitude d'Élisabeth envers les chatons : elle les tend au voisin avec un sourire (63). On ne sait pas si elle utilise cette activité comme une stratégie cathartique pour se débarrasser de sa rage contre le village, mais on peut constater que cette cruauté volontaire envers les animaux ne facilite pas la représentation de la pureté de l'homme. Ainsi, en général, les animaux sont des personnages innocents qui font ressortir la malice des humains, soit sous la forme de violence irrationnelle, soit sous la forme d'égoïsme 
intéressé.

Si l'animal n'est pas employé dans une œuvre en ayant pour fonction de représenter l'innocence ou de faire ressortir clairement les qualités moins pures des humains, l'animal peut fonctionner comme un symbole. Après tout, Jonathan Burt, dans son analyse de Why Look at Animals? de John Berger, confirme : «animals are [...] mapped onto a language of signs; they are 'messengers', 'promises', the 'first metaphor', the 'first symbols', they 'offered explanations' »(207). Il n'est pas surprenant que la facette symbolique antique de l'animal continue d'être employée aujourd'hui sous la forme d'un totem métaphorique. Le dictionnaire Oxford English Dictionary définit ce mot en anglais de cette manière : «[An] animal that is believed by a particular society to have spiritual significance and that is adopted by it as an emblem ». Après tout, les animaux sont souvent associés à des traits et caractéristiques spécifiques, comme le renard connu pour sa ruse et adopté par le personnage principal dans $L a$ rage (Hamelin 26). Ainsi, les totems dans les œuvres peuvent prêter certains traits d'animalité à l'être humain — des traits qui révèlent surtout l'avidité, la colère, la peur, l'instinct « combatfuite », le désir et la violence humaine.

Pour le père de Christine, le cochon propose un excellent exemple de totem dans La rage. Christine baptise son père «Cœur-de-cochon» (235), puisque les docteurs lui ont donné « une valve de porc » (232). Cependant, même si Christine n'est pas consciente de ce fait, ce surnom n'a pas seulement une signification littérale : il joue aussi un rôle symbolique. En rappelant l'image du cochon, qui représente traditionnellement l'avidité, et qui, selon le Larousse de Poche, est aussi un adjectif dénotant une personne «malfaisante » ou « obscène », voire avide de sexe, le nom « Cour-de-cochon » décrit bien le père qui maltraite sa fille de façon perverse.

Parfois, la signification des animaux totémiques peut représenter les émotions et les sentiments négatifs d'une société ou d'un peuple. Dans La rage, Hamelin utilise trois animaux totémiques canins - le chien, le renard et le loup — ainsi que leur rage symptomatique pour symboliser et faire ressortir « le maquis 'rabique' de Saint-Canut » (Grigorut 20). Cette rage peut être interprétée soit comme la colère, soit comme la folie. Dans ce roman, le cheval de trait est aussi employé comme animal totémique métaphorique. Selon les observations de Malarmé qui le voit «avec le moufle fourré dans la terre (Hamelin 290), l'animal semble non seulement enragé comme les villageois à Mirabel, mais aussi désespéré et incapable de se libérer de sa clôture. Cet animal représente donc la frustration affolée des gens de Mirabel, provoquée par leur 
expropriation pendant les années 1970.

De façon similaire, dans La héronnière, Tremblay représente les émotions des gens au village. Ils ne s'y sentent pas contents sinon inquiétés par l'économie et les menaces étrangères (surtout les touristes). Les oiseaux du village sont les animaux totémiques des habitants et représentent bien leur désir de partir pour survivre. Après tout, dans la littérature, les oiseaux sont caractérisés par la migration, « le voyage qui dépayse et qui fait rêver » d'un avenir meilleur (Charbonneau-Hellot 7). De plus, les oiseaux peuvent aussi représenter les femmes telles que Martine et Nicole qui quittent le village inopinément pour protéger leur avenir peu prometteur. L'oiseau est donc, comme le dit Marie-Christiane Charbonneau-Hellot, « un écho de ce vieux rêve de liberté si cher à l'homme » (5) et représente aussi nos tendances à partir quand le danger est présent.

Cette réaction «de lutte ou de fuite » est certainement un instinct animal — et si l'on ne part pas, on se bat, comme dans La rage, surtout avec le personnage de Christine qui reprend les traits totémiques du renard. Par exemple, pour se protéger quand Malarmé essaie de la séduire, Christine «se [contracte] violemment » et elle plante «ses ongles », c'est-à-dire ses griffes, dans le dos de l'homme et le mord au bras, de façon «enragée » et rabique (Hamelin 249). Blessé, Malarmé note que le visage de Christine «[n'exprime] pas de fureur, simplement une volonté animale de résistance farouche » (249). Quand la proposition d'Édouard de faire l'amour à Christine se répète, Christine réagit en poussant un cri «fort et aigu » et en mordant le cou d'Édouard (289). Ce dernier est mordu encore une fois plus tard dans le roman, au même endroit, par un vrai renard (351). Ainsi, les animaux totémiques ne sont pas limités aux groupes de personnes : ils peuvent aussi représenter des individus.

Le protagoniste de La rage représente l'individu qui exemplifie le trait le plus « animal », c'est-à-dire le désir sexuel, puisque le terme «animal » l'évoque du fait de son association au phénomène de la reproduction, motivation et instinct primaire pour les créatures de la planète. Dans le roman, Malarmé voit un faisan qui lui échappe et le nomme ainsi «un faisan de Christine » (295), car à la manière de Christine qui échappe à Malarmé, le faisan prend son envol. Néanmoins, cet oiseau est significatif : bien que Malarmé adopte le renard comme totem dans le roman, l'oiseau devient son deuxième totem dans cette scène-ci. Selon Malarmé, l'oiseau est son serviteur annonçant « une expansion spatiale à [sa] prise de possession » et le couronnant pour faire de lui le « roi de la chasse » (296). La chasse, dans ce cas, implique la chasse de 
Christine. Autrement dit, le faisan, symbole traditionnel de la libido et représentation du désir sexuel selon le guide des animaux spirituels du praticien chamanique Steven Farmer (209), rappelle à Malarmé son désir de posséder et de séduire Christine. Ainsi, l'animal totémique souligne le désir animalesque de l'humain. Enfin, le dramaturge Michel Marc Bouchard considère le symbolisme des animaux pour les employer comme des totems dans la pièce Tom à la ferme. Francis, le frère du défunt, est fasciné par leur présence, indiquée par les jappements d'un chien local (Bouchard 33). Dans cette pièce, il «parle [souvent du] coyote» (33), son animal totem, alors que le lièvre devient le totem de Tom, qui l'adopte en affirmant qu'il est luimême « comme un lièvre » (36).

Les animaux totémiques de Tom à la ferme reflètent les personnages et leurs caractéristiques : dans le règne animal, le coyote domine le lièvre, qui est sa proie naturelle. Cette domination s'observe lorsque Francis raconte, de façon fascinée et subjuguée, l'histoire où ses voisins ont observé un coyote : «[il y] avait un [coyote] qui s'amusait avec un lièvre, [qui n'était] pas encore mort. Il lui donnait des coups de pattes pour le faire bouger. Le lièvre essayait de s'enfuir. Le coyote le rattrapait, le maganait, le laissait partir, le rattrapait » (34). Ce passage, qui décrit le comportement tyrannique du coyote, ressemble à celui de Francis quand il intimide Tom avec violence tout au long de la pièce. On peut voir ce comportement dans les didascalies lorsque Francis «prend Tom tout contre lui comme on fait avec un animal domestique » (52). Ici, on peut attribuer la soumission de Tom à une manifestation du phénomène bestial du mâle alpha qui établit des hiérarchies entre dominants et dominés dans le règne animal. Ainsi, les totems de Francis et de Tom leur prêtent respectivement des traits violents et passifs.

Néanmoins, la manifestation de l'animalité la plus évidente dans la pièce est sans doute la scène du septième tableau où Tom, terrifié, déclare qu'il « [n'est] pas humain » (54). En avouant qu'il est plutôt un «morceau de viande au-dessus de la charogne » et «juste un repas [pour les coyotes]»(54), il se déclare la proie de Francis, qui adopte la passion pour le harcèlement tyrannique du coyote en «[imitant] le grognement [de l'animal] » et en s'amusant du fait que Tom est suspendu au bout de sa corde, bien que Tom lui demande de pouvoir sortir (54). Cette scène, qui illustre parfaitement la manifestation des caractéristiques des animaux totémiques chez les personnages, démontre la violence animalesque que les humains peuvent présenter. Selon MacDonald, dans le programme sauvage des animaux, il «y est question de survie et de l'amour et d'appétit et de besoins et de plaisir et de peur. En d'autres mots, d'appétence totale ». 
En interprétant les animaux des textes littéraires comme des totems qui animent et font ressortir nos qualités d'animalité, on peut se demander ce que cela signifie à propos de la supériorité de l'humain. C'est peut-être une simple indication que les êtres humains sont aussi «méchants » que les animaux qu'ils conçoivent ainsi.

En conclusion, les tableaux de chasse de Rubens, les peintures hyperréalistes de Colville, le roman La rage de Hamelin, la pièce de théâtre Tom à la ferme de Bouchard et les nouvelles de La héronnière de Tremblay montrent le rôle important que l'animal joue dans nos œuvres artistiques et littéraires. Comme maître et gardien, l'animal souligne notre impuissance physique. En fonctionnant comme un personnage innocent abusé, il fait ressortir l'égoïsme, la cruauté et la malice de l'être humain - ou parfois comme un totem qui souligne des traits d'animalité. L'animal dans ces œuvres montre que l'humain n'est pas forcément plus raffiné ni avancé que la bête. L'humain est souvent plein de malice et d'animalité. L'être humain condamne les traits négatifs chez les animaux, mais dans ces œuvres, ceux-ci sont employés en contrepoint du genre humain. Ils font ressortir les aspects de l'animalité de l'être humain et signalent les traits qu'ils ont en commun. En réalité, l'être humain admire l'animal tout en étant aussi animalesque - ou plutôt pire. Ces accusations sont donc pleines d'hypocrisie. Mais l'illustration d'animaux en littérature comme en peinture relève d'une certaine reconnaissance de la part des êtres humains envers ces derniers puisqu'ils représentent des modèles à honorer et à imiter.

\section{Bibliographie}

«Animaux - Alex Colville - Musée des beaux-arts du Canada ». Musée des beaux-arts du Canada, Musée des beaux-arts du Canada, 2015. http://www.beauxarts.ca/colville/fr/23.htm

Bouchard, Michel Marc. Tom à la ferme. Montréal : Leméac, 2011.

Burt, Johnathan. «John Berger's 'Why Look at Animals ?': A Close Reading ». Worldviews : Global Religions, Culture, and Ecology. 9.2 (2009) : 203-218.

Berger, John. Ways of Seeing. Londres : Penguin UK, 1972.

---. Why Look at Animals ? Londres : Penguin UK, 2009.

«Pierre Paul Rubens : la Chasse au tigre ». Réalisé par Fabrice Hourlier, proposé par Nathalie Boels et Tim Newman, présentation Frédéric Taddeï, production Fouzia Kechkech. D'art d'art. Froggies. 2006. 
Charbonneau-Hellot, Marie-Christiane. «L'enfant et la bête : le visage et la fonction des animaux dans la littérature pour la jeunesse au Québec de 1979 à 1982 ». Lurelu 6.3 (1984) : 3-11.

Cohen, Simona. Animals as Disguised Symbols in Renaissance Art. Leiden / Koninklijke : Brill, 2008.

Farmer, Steven. Pocket Guide to Spirit Animals. New York : Hay, 2012.

Grigorut, Constantin. «Un mythe voyageur et son ancrage intertextuel dans le roman La rage de Louis Hamelin ». Nouvelles études francophones 15.1 (2000) : 17-32.

Hamelin, Louis. La rage. Montréal : XYZ, 1995.

MacDonald, Ann-Marie. «Alex Colville au Musée des beaux-arts du Canada: Ann-Marie MacDonald ». YouTube, téléchargé par le Musée des beaux-arts du Canada, 17 fév. 2015. https://www.youtube.com/watch?v=AAFJg4tKMVE.

Paré, Denise. « Habitats, migrations et prédations : Analyse écocritique de La héronnière de Lise Tremblay ». Cahiers de géographie du Québec 52147 (2008) : 453-470.

Tremblay, Lise. La héronnière. Montréal : Leméac, 2003. 